\title{
Investigating User Attention and Interest in Websites
}

\author{
Alistair Sutcliffe $^{1}$ and Abdallah Namoune ${ }^{2}$ \\ Centre for HCI Design, School of Informatics, University of Manchester, UK \\ alistair.g.sutcliffe@manchester.ac.uk, \\ abdellah.namoune@postgrad.manchester.ac.uk
}

\begin{abstract}
Users' attention was investigated by eye tracking, combined with reported rating of areas of interest, and free recall memory of six operational websites. The sites differed in the pattern of fixations recorded depending on their layout structure. Fixation durations and areas of interest were generally correlated but exceptions were present in both directions. The sites which were rated more attractive overall had an open layout and high density fixations on animations. The sites which were preferred overall had column layout, and content with brand seemed to be the more important determinants for preference. Fixation densities were closely related to reported user interest for $4 / 6$ sites but not for two e-commerce sites. Reported attention, positive memory and overall preference were weakly related.
\end{abstract}

Keywords: Eye tracking, visual attention, website evaluation.

\section{Introduction}

Eye tracking studies of websites have been popular as a means of diagnosing the effectiveness of designs, for instance in comparing optimal navigation pathways and user attention patterns as revealed by eye tracking pathways [9]. Eye tracking, either using sequence analysis or overall fixation durations and densities, is being used as a diagnostic tool to evaluate designs by comparing patterns of fixation with areas of interest on websites; for example, an F-shaped pattern has been proposed as evidence of good design [13]. Eye tracking studies have indicated influences on users' attention, such as animation having a dominant influence on directing fixations [4] and the influence of the cursor's locus on users' attention [17]. In a website study, users' reported attention from concurrent protocols was compared with eye tracked fixations showing that $70 \%$ of the reported areas of interest were also fixated [12]. However, what users attend to and what they look at may not be so closely correlated; for instance, Burke et al. [3] have demonstrated that saccades and limited fixations in the proximity of objects may be sufficient for cognitive processing, and that banner adverts are not always fixated and can be ignored.

Fixations as revealed by eye tracking studies could be determined by image salience and goal-directed attention [10,11]; however, objects that are actively processed by users may not be the same as those that are fixated. Therefore we investigate the link between fixations, users' perceived attention to objects of interest, their overall preference and memory. The paper is organised as follows. A review of 
related work is followed by a description of the selected websites and the experimental methods. Then the results of eye tracking analysis, users' perceived attention, and overall preference ratings are described. Finally the discussion reviews the contributions of the study and implications for future use of eye tracking as an evaluation tool.

\section{Related Work}

Eye tracking studies have investigated how people read news web pages [14], suggesting that the first entry point for most web pages is located in the upper left corner and is usually a headline. Animation has been considered to have a dominant effect on user attentions and directing fixations [4,5]; however, in studies on banner adverts in web pages, no significant effect was found on the ability to recall and recognise banner ads [1]. Burke et al. [3] found that banner ads distracted the users' visual search and significantly increased their search time; furthermore, memory recall for animated banners was worse than for static banners.

Zhang instructed participants to identify and count text strings on a web page that contained an animated distractor [18]. She found that animations decreased user performance while searching for information and the effect of animation on performance was determined by the complexity of the task. The more similar an animation was to the task, the worse user performance became; brightly coloured animations interfered more than dull coloured animations. Eyetracking sequences are influenced by the layout and density of displays [7,8]; however, the relationship between fixation sequences and densities and users' attention is less clear.

Eye tracking studies [15] on 11 websites in four categories - shopping, business, search and news - showed that the nature of the task (browse/search) did not significantly influence the fixation patterns, although gender, the viewing order of web pages, and the interaction between page order and site type influenced user eye movements. Guan et al. [6] investigated the association between eye tracked fixations and user attention reported in a retrospective protocol for problem-solving tasks using graphical displays. Agreement between the reported and fixated areas was modest (53\%); in contrast, better agreement (70\%) was found by Johansen and Hansen [12] who used concurrent protocols.

\section{Materials and Methods}

Six websites (see Figure 1) were chosen to represent a diverse set of applications and types of design. The sites were selected to investigate exploratory hypotheses associated with two or more of the sites.

1. Fixation patterns will be influenced by the structure and layout of the site.

2. Animations and images of people will receive high fixation frequencies, as suggested by the computer as a social actor paradigm [16].

3. Areas of high fixation durations/frequencies will be correlated with users' reported areas of interest.

4. Sites with more frequent fixations and reported interest will invoke more detailed memory. 


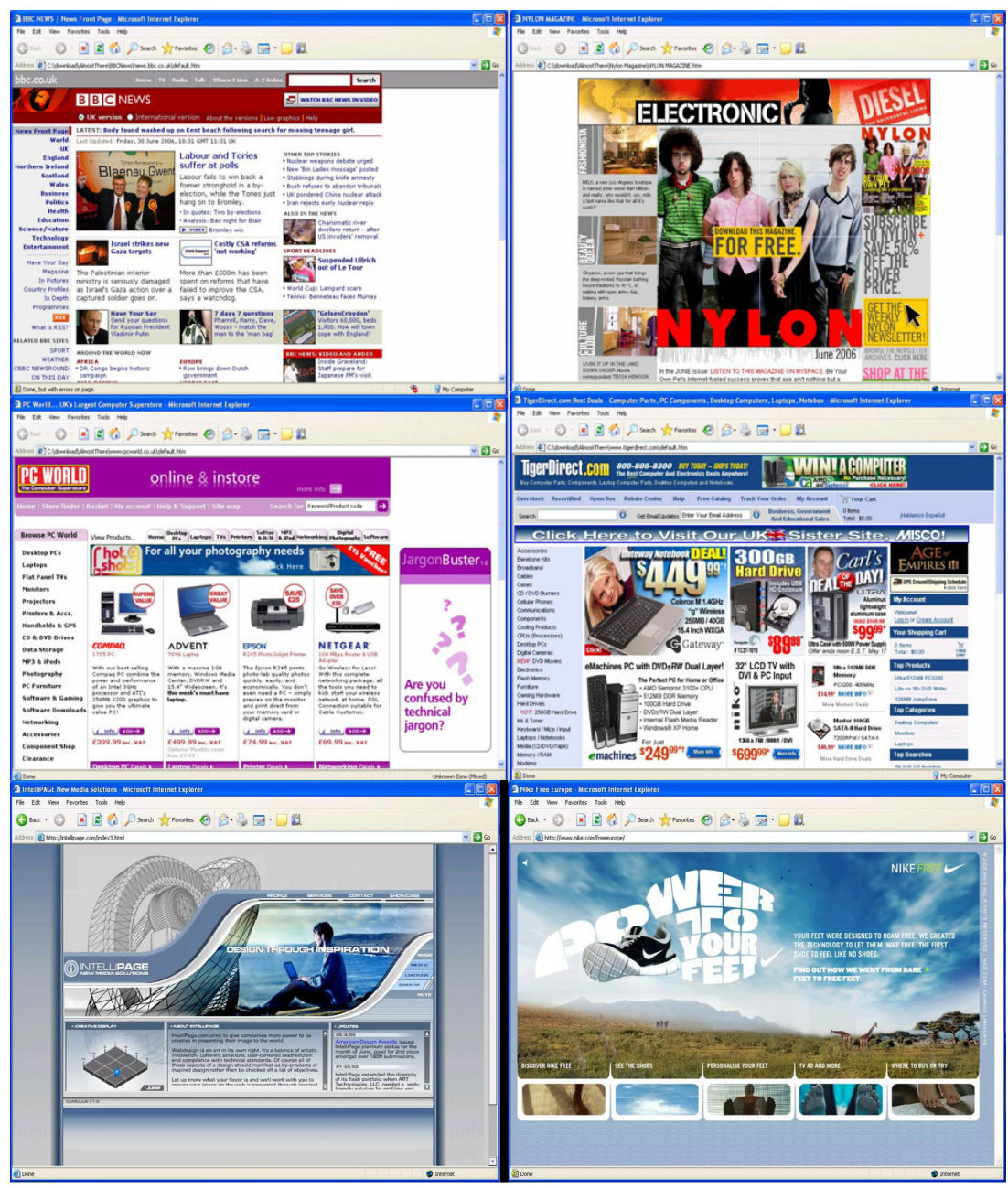

Fig. 1. BBCNews (upper left), Nylon (upper right), PCWorld (middle left), TigerDirect (middle right), IntelliPage (lower left) and Nike (lower right)

The hypotheses were posed to explore associations between users' attention, their perception of interesting design features and content, memory for features and content, and their overall rating for the site. The following sites were selected:

BBCNews: an information provider site with a strong brand image. The BBCNews site follows a traditional columnar block structure. This site had small animations in the centre above the top stories section.

Nylon: an information provider. Nylon magazine has similar goals to BBCNews, portraying interesting content to the user with good navigation to facilitate exploration of content. Animation was used on the central image. 
PCWorld: an e-commerce site retailing computers and related technology. This site had a traditional block-structured column layout. Animated banner adverts were used in the central column.

TigerDirect: an e-commerce site retailing computers as a direct competitor to PCWorld, although with a less well known brand. One banner advert was present at the top of the page.

IntelliPage: an information provider that also sells design services. It has an open design format. Animation was used on the main central image with two concurrently running effects.

Nike: an e-commerce site with a strong brand image and design emphasis. It made extensive use of animation and graphics and had a more open design format.

Thirty subjects (15 males, 15 females, mean age 24 , range 18-46 years) who were students and researchers at the University of Manchester took part in the investigation. Most subjects (26/30) had 3 years or more Internet experience, and all used the Internet daily or at least once a week. The subjects were familiar with the BBCNews website (68\% had visited it $>5$ times) and some were aware of the PC World site (33\% had visited it 2-5 times); while only 3 had visited Nike 2-5 times. None of the other sites were known to the subjects. The experimental procedure consisted of the following steps.

(a) The participants completed a pre-test questionnaire recording their Internet experience, whether they had viewed any of the sites, and their interest in the subject matter of the sites.

(b) The participants were asked to view six different web pages for 60 seconds on a 17 " monitor with screen resolution set to $1024 * 768$ pixels. User eye movements were recorded with a Tobii 1750 eye tracker. The number of fixations, duration of fixations, and dwell time by screen area (heat map) were analysed. The participants were instructed to browse the page for any items that might interest them.

(c) They completed a free recall memory test by listing any objects or areas on each site that they could remember, and rating each item as either positive (liked), neutral or negative (disliked).

(d) They then recorded their perceived attention to different areas on each site by marking the areas on a screen dump image. They ranked the areas of the screen by order of interest.

(e) The participants rated each site on a questionnaire capturing their preference for the sites, taking their purpose into account, and overall rating of the website's attractiveness in terms of design quality.

The order in which the home pages were presented was counter-balanced. Every user was first asked to freely browse the home page of each site for 60 seconds. Participants were explicitly instructed not to click on links within the pages; however, they could freely scroll down to view all the area of the page. After viewing all web pages, the subjects re-viewed the home pages and reported their sequence of attention by pointing to and verbally describing areas on-screen. They then specified and ranked areas of interest by pointing to areas on a print-out of the home page. Web 
pages were divided into areas of interest according to their structure and media, e.g. menus, text boxes, images, animations, link panels and logo, to enable the subjects' reports to be classified. Subjects were paid $£ 10$ for the experiment, which concluded with a debriefing interview to elicit their opinions about the designs, features that interested them, reasons for preferring any particular design, and criticism of design quality.

\section{Results}

\subsection{Eye Tracking Analysis}

There were no differences in the overall fixation duration between the sites, which received between 46.5 and 47.1 secs fixation out of the total measured period of 55 seconds. However, the total number of fixations (defined as foveal focus held in a constant location $>200 \mathrm{~ms})$ did differ between the sites $(\mathrm{F}=7.05$, df $5, \mathrm{p}<0.001)$ with means ranging from 163 (BBCNews, Nylon and TigerDirect) to 162 (PCWorld), 155 (Nike) and 146 (IntelliPage). Sites with column block structures (BBCNews, PCWorld, TigerDirect, and Nylon - see Figure 2) had heat map patterns that followed the columns, with more attention being paid to the top of the page. For BBCNews users, viewed the lead stories in the middle and right hand columns with associated images and text in the adjacent columns. In PCWorld, users fixated on the products following each of the four columns, and a similar pattern occurred in TigerDirect with a more diffuse pattern of fixations. Nylon showed an intermediate pattern since it had a large prominent centrally located image which attracted users' attention with the text located beneath it. The subjects also fixated on areas following the column layout.

Nike and IntelliPage had a different pattern, which reflected their non-columnar layout. Users' fixations were focused on animations and salient images without any evidence of a layout order; furthermore, the number of distinct fixated objects/areas was lower. In these sites users' attention seemed to be located on one prime area with 3-4 sub-areas.

The areas fixated showed a strong influence for animation in three sites: Nike, IntelliPage and Nylon; the remaining sites showed less effect, even though they did have animations and banner adverts. This appears to confirm previous findings of users selectively ignoring banner adverts, while interesting animations receive considerable attention.

The sequence of fixations was analysed by dividing each screen into $0.5 \mathrm{~cm}$ cells to plot the locus of fixations. A common fixation path for all subjects was calculated by starting with the cell with the highest first fixation count, then taking the cell with the highest frequency of second fixations, and so on. The first 15 fixations in the most frequent surviving pathway were analysed, which accounted for approximately 10-15 secs duration. Most pathways started with $>50 \%$ of the users, but branching reduced the commonality for nodes later in the sequence. Fixation sequences are shown in figure $3 \mathrm{a}-\mathrm{c}$ with the square box outline, circled numbers and solo numbers refer to 


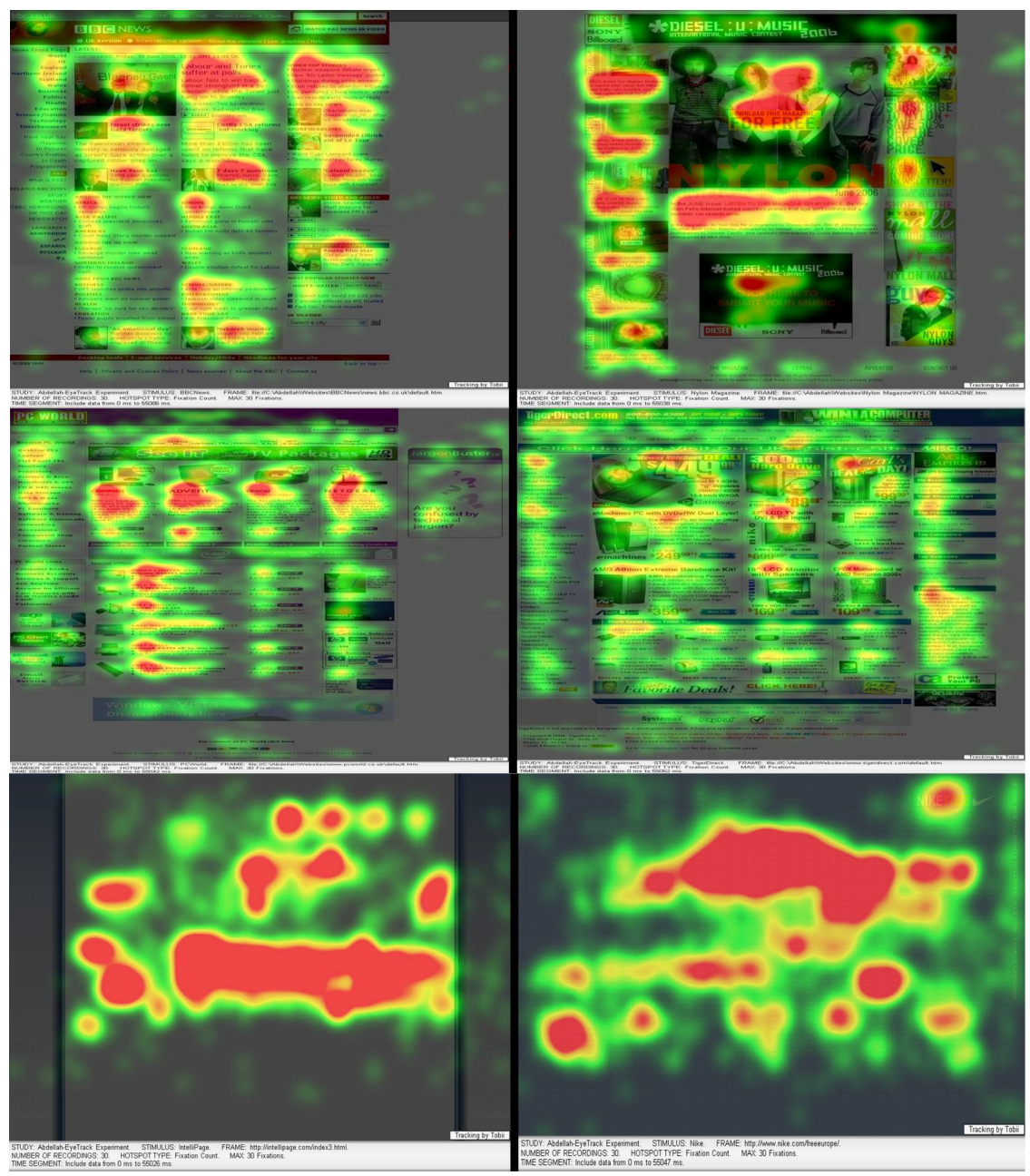

Fig. 2. Heat maps showing distribution of fixation densities for BBCNews (upper left), Nylon Magazine (upper right), PCWorld (middle left), TigerDirect (middle right), IntelliPage (lower left) and Nike (lower right)

high ranked reported areas of interest and areas with high total fixations (explained in section 4.2). In the BBC site (see Figure 3a) initial fixations follow the main news stories, with some attention (13-15) to an animation. Early fixations in the Nylon site were on the central image which was animated, with later ones on the news stories. For PCWorld and Tiger Direct the sequence suggests the users scan the content of the sites in the top menu bars and left hand menu in PC World, and the products on the top part of the screen. In contrast to other sites, animations and banner adverts did not attract fixations in the early sequence. The sequence observed in the Nike site did follow the appearance of animations, while the Intellipage sequence was also located by animated areas on the screen, although the sequence was more erratic since the 
animations started concurrently. Generally many of the areas which were fixated in the initial sequence do not appear in the top interest or total fixation ranked order. If animations are present they appear to determine the fixation sequence, although this was not true for PCWorld and TigerDirect where products and site content seem to have been more dominant.

\subsection{Perceived Attention}

Users' reported attention to areas on each site was compared to their eye tracked fixation durations. Each site was divided into areas based on its structure and functionality, i.e. segmented display areas, menus, images, animated areas, link lists, etc. The subjects' reported interests were mapped to these potential areas of interest. Reported areas of interest (AOIs) were ranked by calculating the average interest rating for each area multiplied by the \% of users who rated that area. Reported AOIs were compared with higher fixation densities by dividing the screens into $0.5 \mathrm{~cm}$ cells and then comparing cells with fixation densities $>1 \%$ total fixations with the heat maps, and the users' retrospectively reported areas of interest; see Table 1 . Where the boundaries of fixation cells and reported areas showed partial overlapping, agreement was scored as 0.5 .

Table 1. Agreement between reported areas of interest and high density fixation areas

\begin{tabular}{lccccc}
\hline Site & $\begin{array}{c}\text { Potential } \\
\text { total } \\
\text { AOIs }\end{array}$ & $\begin{array}{c}\text { Reported } \\
\text { areas \% } \\
\text { of total }\end{array}$ & $\begin{array}{c}\text { Fixated } \\
\text { areas \% } \\
\text { of total }\end{array}$ & $\begin{array}{c}\text { Fixated and } \\
\text { Reported } \\
\% \text { of } \\
\text { Reported }\end{array}$ & $\begin{array}{c}\text { Animations } \\
\text { Fixed and } \\
\text { Reported }\end{array}$ \\
BBC & 18 & 83 & 66 & 80 & yes \\
Nylon & 8 & 100 & 77 & 77 & yes \\
PCWorld & 19 & 84 & 26 & 31 & no \\
TigerDirect & 23 & 87 & 35 & 40 & no \\
IntelliPage & 8 & 100 & 94 & 94 & yes \\
Nike & 7 & 100 & 76 & 76 & yes \\
\hline
\end{tabular}

In all sites most potential AOIs were attended to from the users' reported evidence, and, apart from PCWorld and TigerDirect, there was close agreement between reported and fixated areas. The animations in close agreement sites were also fixated and reported. No areas were fixated but not subsequently reported as interesting. And all areas containing images of people were reported as interesting and fixated. However, from debriefing interviews only the images of people on IntelliPage (man sitting), in the central Nylon image, and the woman in the TigerDirect advert were commented upon, so small images may have been attended to for their associated text. The lower $\%$ of fixations in TigerDirect and PCWorld may be an artefact of the retrospective reporting. These sites had more complex structures and hence higher potential AOIs. The subjects cited interest in 
most areas, whereas their fixations were concentrated on a few areas where products were displayed. We conjecture that subjects' reported interest was 'reconstructed memory' based on their expectations of e-commerce sites; in contrast, the BBC site, which also had a complex structure, showed better agreement between reported interest and fixations. Debriefing comments suggest this may be due to subjects' interest in the variety of news stories.

The top five areas measured by fixation densities for each site (indicated by numbers) and the user-reported top five interest areas (numbers in circles) are shown in Figures 3a-c. Overall there was considerable agreement between the fixation densities and subjective ratings of areas of interest, apart from PCWorld and TigerDirect. However, when the top five fixation densities and reported AOIs are used as a measure of salience, in all sites there were 1-2 areas which were reported but not fixated, or fixated but not in the reported top five. The rank ordering for fixation densities and reported AOIs in each site were also different. In the BBCNews site, for example, several users reported the left area story and BBCNews video and newsround stories as interesting, even though they did not fixate on them frequently. Conversely, users fixated on other stories and the around the world section, which were not subsequently rated as interesting. This may reflect a scanning strategy to sample items which are later discarded.

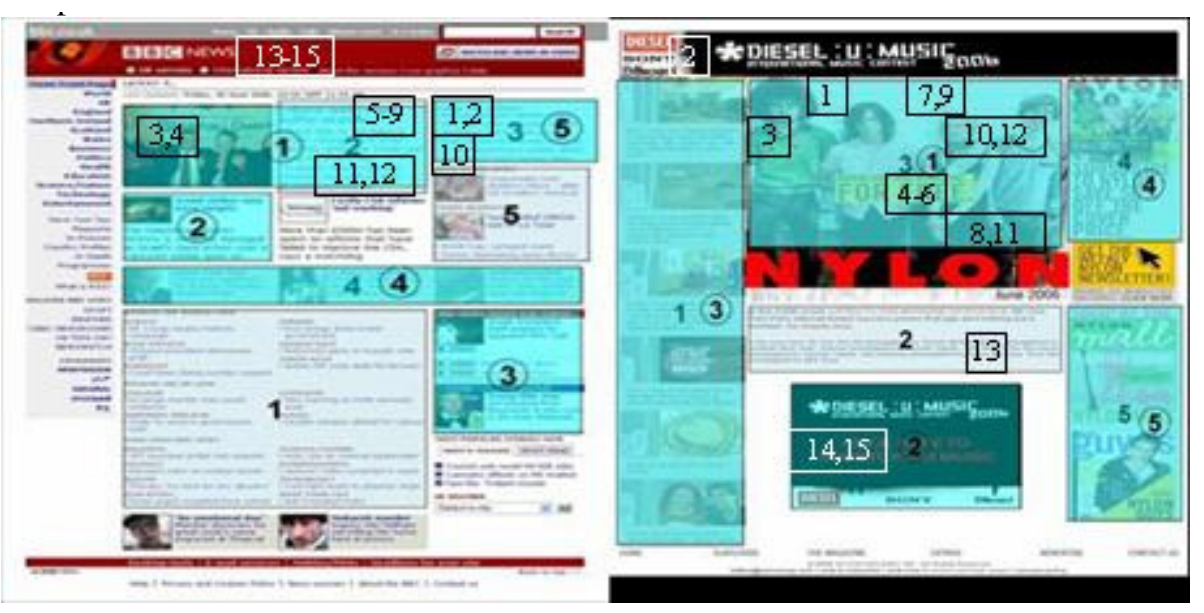

Fig. 3a. User-reported attention to top five areas of interest (number + circle) and top five fixation densities for BBCNews (left) and Nylon (right)

Animations in the centre of the page attracted user attention in the Nylon page and were rated as interesting. One item in the interest list (bottom animation) was not fixated, while the centre text was fixated but not rated as interesting. In this case, it appears that users may read the text from fixation evidence but then relegate it to lower down their interest list. 


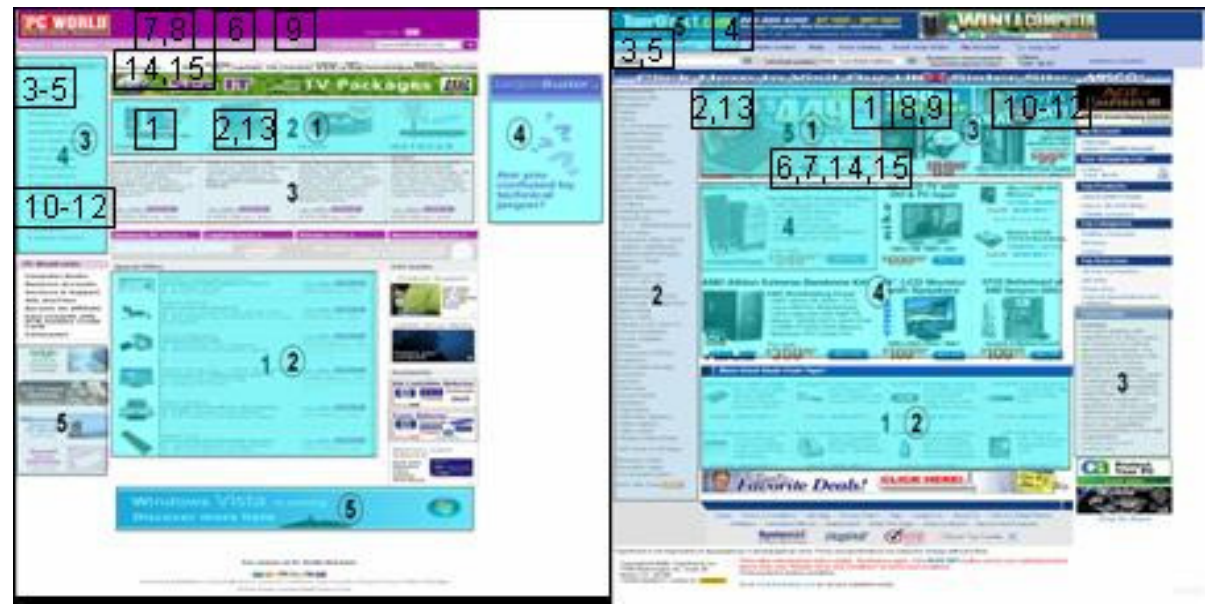

Fig. 3b. User-reported top five areas of interest and top five fixation densities for PCWorld (left) and TigerDirect (right)

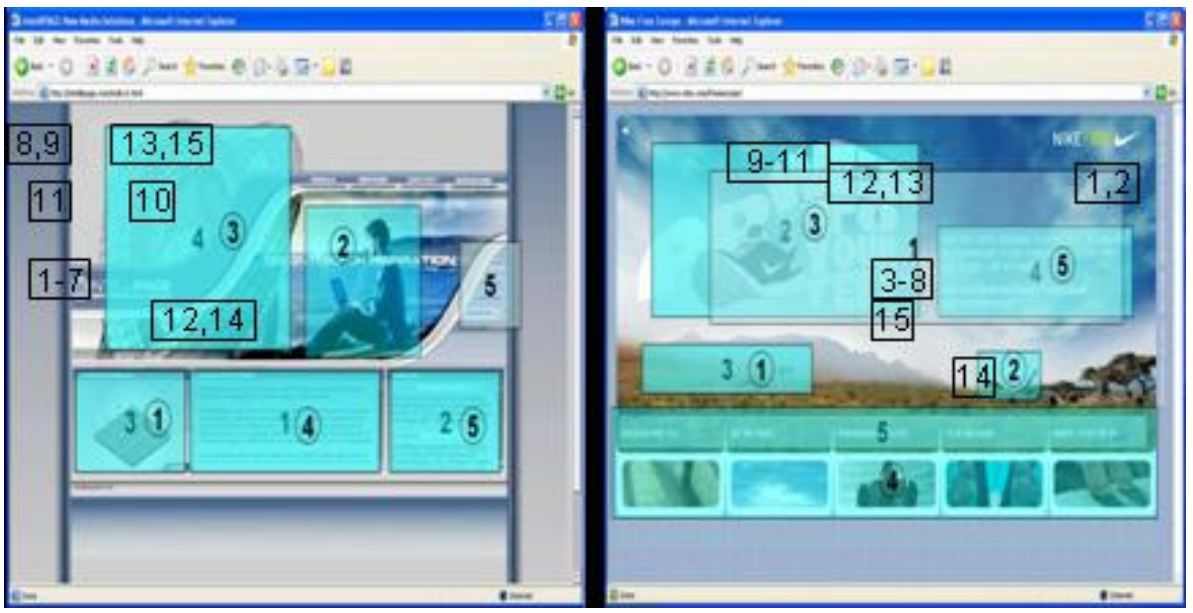

Fig. 3c. User-reported attention to top five areas of interest and top five fixation densities for IntelliPage (left) and Nike (right)

PCWorld and TigerDirect showed a weaker correspondence between the subjective and objective measures, which may reflect a user strategy of scanning these sites, since the heat maps also showed a less dense, more distributed pattern. Images of products in the centre of the page attracted attention in PCWorld and TigerDirect, followed by product images lower down the page. However, banner adverts in both sites were only partially effective. In these block-structured sites, users fixated on the products but tended to ignore the adverts. In their subjective record they reported interest in only a sub-set of the areas they fixated on, so it appears that they were selectively ignoring some areas. 
Subjective and objective attention was in close agreement for Nike apart from the second area of interest (giraffe animation) which was reported as interesting but not fixated. In Nike the order of fixation and interest were linked to the unfolding sequence of animations (text, man, giraffe) which led the user towards the menu for product choice and purchasing. Some parts of the IntelliPage animation were fixated but ranked as less interesting. The sequence of animations in Nike followed in a smooth order, whereas the IntelliPage animations ran concurrently and competed with each other. For IntelliPage, both subjective attention and objective measures agreed, apart from area 2 in the interest ranking (man on beach image) which was not highly ranked in fixation density. The heat map shows this area was fixated but not intensely, so it appears that users may register areas of interest from less frequent fixations. Open structure sites (Nike, IntelliPage) showed a stronger correspondence between fixations and reported areas of interest. Nylon appeared to follow an intermediate pattern, probably reflecting its columnar plus large central image hybrid design.

\subsection{Memory}

The sites differed significantly in the total number of items remembered $(\mathrm{F}=2.74$, df $5, \mathrm{p}<0.05)$ and rating valency $(\mathrm{F} 9.93$, df 5, $\mathrm{p}<0.001)$; see Table 2.

Table 2. Memory for each site, total items and likeability weighted by valency. Scoring: positive items +1 , negative items -1 .

\begin{tabular}{l|c|c|c|c}
\hline & Total & \% Content & $\begin{array}{c}\text { Likeability } \\
\text { weighted }\end{array}$ & $\begin{array}{c}\text { \% } \\
\text { liked/total }\end{array}$ \\
\hline BBCNews & 166 & 56 & 91 & 55 \\
\hline IntelliPage & 159 & 53 & 35 & 22 \\
\hline Nike & 188 & 28 & 116 & 62 \\
\hline Nylon & 150 & 33 & 48 & 32 \\
\hline PC World & 177 & 37 & 58 & 33 \\
\hline Tiger Direct & 174 & 63 & 19 & 11 \\
\hline
\end{tabular}

Subjects remembered more items overall and more positively rated items for the Nike site. The differences were significant with all sites except BBCNews (T-tests, $\mathrm{p}<0.05)$. However, Nike's content was not so well remembered; instead, users remembered the animations (52\% of all items). TigerDirect was remembered well in overall volume, but rated much lower in likeability. A higher proportion of content items were remembered for TigerDirect than for PCWorld, which might be explained by the more diffuse heat map pattern indicating that users scanned this site more completely. Also, the larger images used by TigerDirect may have stimulated users' interest more effectively. The BBCNews site had the second highest volume and liked memorised items, and most of these were content related, so it appears that while BBCNews was remembered for its content and brand, Nike was remembered for its design. Nylon produced more animation-related memory (39\%) whereas, 
unexpectedly, IntelliPage which had an animation-intensive design produced more content memory, but had low likeability ratings. Debriefing comments indicated that users didn't like the animations although they remembered their content (e.g. the pin ball animation) adversely. When the top five reported-interest areas were compared with memorised items, the animations in Nike, IntelliPage and Nylon were in close agreement; BBC content memory and reported areas also agreed, while agreement was poor for PCWorld and TigerDirect apart from general memory for products.

\subsection{Overall Preference}

The sites were significantly different in overall preference $(\mathrm{F}=25.28$, df $5, \mathrm{p}<0.001)$ with BBCNews and Nike being the most favoured sites. For attractiveness the difference between sites was also significant $(F=48.72$, df $5, p<0.001)$. For overall preference, BBCNews and Nike were the most favoured websites, followed by Nylon, IntelliPage and PCWorld close together, with TigerDirect in last place. The preference ratings of $\mathrm{BBCNews}$ and Nike were significantly higher than in the remaining websites $(\mathrm{p}<0.01)$. Nike was perceived to be the most attractive website $(\mathrm{p}<0.001$ on overall measure), followed by Nylon and IntelliPage, with BBCNews in fourth place. TigerDirect scored significantly lower than all other websites in terms of preference and attractiveness $(\mathrm{p}<0.01)$. Table 3 summarises the overall preference and attractiveness weighted scores for each website.

Table 3. Mean weighted ratings for overall preference and attractiveness (ranks 1-5 weighted by $0.5,0.25,0.12,0.05,0.03,0.02$ )

\begin{tabular}{l|c|c|c|c}
\hline & \multicolumn{2}{|c|}{ Preference } & \multicolumn{2}{c}{ Attractiveness } \\
\hline & $\mathrm{N}^{\text {st }}$ choice & Overall & $\mathrm{N} \mathrm{1^{ \text {st } }}$ choice & Overall \\
\hline BBCNews & 15 & 9.85 & 1 & 3.86 \\
IntelliPage & 2 & 3.04 & 2 & 4.73 \\
Nike & 10 & 8.98 & 20 & 12.30 \\
Nylon Mag & 1 & 3.43 & 6 & 6.11 \\
PCWorld & 1 & 3.16 & 1 & 2.19 \\
TigerDirect & 1 & 1.54 & 0 & 0.81 \\
\hline
\end{tabular}

There were no significant correlations between measures of attention (total fixations, fixation duration) and overall preference and attractiveness. Valenced memory (likeability) was correlated with attractiveness $(\mathrm{p}=<0.001$ Spearman $\mathrm{r})$, but not preference. Total memory was not correlated with preference or attractiveness.

In debriefing interview comments, BBCNews was preferred overall for its content, but was deemed to be less attractive; in contrast, Nike was the most attractive site and this corresponded with the attention measures and memory. The Nylon site also received a good attractiveness rating which is consistent with the users' memory. The anomaly is IntelliPage which ostensibly had good graphical design and creative use of animation; however, its attractiveness and preference ratings were poor and this was consistent with the users' memory, giving it fourth place overall. Finally, of the 
e-commerce sites, PCWorld seems to be the more effective design in terms of preference and attractiveness, although TigerDirect produced more items in the memory test. More volume, however, did not appear to be linked to a positive user attitude.

Nike was second on overall preference, while Nylon ranked third, as well as holding second place for attractiveness. PCWorld and TigerDirect occupied the fifth or sixth positions on most measures, apart from total memory where they both scored well. Nike and IntelliPage both attracted users with an interesting design. However, although IntelliPage attracted attention and evoked high content memory, it was not well rated on overall attractiveness. Users appear to have found the design interesting but ultimately unsatisfying. We speculate that the differences in use of animation between IntelliPage and Nike may account for the users' reaction. Nike was a well crafted sequential story, whereas IntelliPage suffered from concurrent overload of multiple animations. We attribute the attractiveness of the Nylon site to its more adventurous use of animation and images.

\section{Discussion}

To revisit our hypotheses: we found strong evidence to confirm that the structure of a website does influence user attention in terms of visual fixation. Reported attention to areas of interest and higher density areas of fixations agreed overall but the order of interest ranking and fixation densities were not related, in agreement with previous studies [12,15]. Structure influences the distribution of fixations and the overall number, with columnar sites receiving more fixations than more open designs. Column structured sites had a more evenly distributed pattern of fixation densities in the heat map analysis, whereas the graphical open structure sites had fewer denser fixations areas. This distribution, and the intermediate pattern in the Nylon site, suggests that image and animation may drive fixation attention when layout structure is not dominant; in contrast, strong layout structure may suppress attention to animations, as we found in the PCWorld and TigerDirect sites. Initial fixation sequences were also driven by animations in most sites, part from PCWorld and TigerDirect were site structure or users' conscious suppression of attention to animated banner adverts may provide the explanation. Initial fixation sequences showed poor agreement over fixation densities and reported interests, so eyetracking pathway analysis may not be useful for evaluating how well site features determine user attention and subsequent interest. We confirmed our second hypothesis that animations and images of people attract attention, both from subjective reports of areas of interest and fixation durations. However, we also found some evidence for banner advert blindness, confirming previous studies $[1,2]$, so while animations might attract, users can override endogenous attention for disliked items. There was little evidence to support our last hypothesis that sites attended to more will be remembered in more detail. There were no differences in reported attention, while the differences in total fixations were associated with site layout structure.

Although most of the frequently fixated areas were also highly rated as interesting by the users, there were exceptions in both directions. We found that reported areas of interest did not always agree with high duration fixations and vice versa, so eye 
tracking may not be reliable for evaluating which key design/content features are attended to. We conjecture that users may discover areas of interest by short fixations or proximal fixations, which agrees with the findings of Halverson and Hornof $[3,8]$. Conversely, high density fixation areas may not be automatically equated with high interest, since users may subsequently downgrade their interest in an area. The interest report measure we used was essentially a cued recall retrospective protocol, so we believe we were capturing users' activated memory for areas of interest. If these areas are positively valenced then such report might be a good predictor of site acceptability and return visits, as demonstrated by our results on memory and attractiveness ranking. Guan et al. [6] also found that retrospective reports of interest reliably agreed with eye tracking fixations; however, our study found a better agreement (circa $70 \%$ v 50\% agreement). This may reflect our use of pointing to AOIs compared to Guan et al.'s indirect mapping of verbally reported objects to image areas. However, the poor agreement we found for the two e-commerce sites shows that retrospective protocols may be prone to reconstructed memory bias; alternatively, low density fixations may be sufficient for the registering the users' interest.

Measures of attention generally were not strongly related to users' memory and overall preference. The lead of BBCNews for preference we attribute partly to the influence of brand and our subjects' prior knowledge, combined with stimulating content. Since we had no task/scenario in this experiment we can tentatively suggest that content and information were dominant in user judgment of information and ecommerce sites (i.e. BBCNews, Nylon, PCWorld and TigerDirect), whereas aesthetics dominated for Nike and IntelliPage. Since content could have had a significant effect, particularly for the BBC site which was familiar to our users, the conclusions on the influences on attractiveness and preferences have to be tentative. In our future work we will refine the methodological approach we have proposed and test sites with a stronger scenario and content assessment to investigate how attention, preference and memory are influenced by users' tasks. Based on the modest association between fixations, reported attention, and memory we found in this study, we expect the value of eye tracking as a diagnostic evaluation instrument may be limited to analysing areas which designers wish to be attended to but which received neither high fixations nor reported attention, or were not remembered.

\section{References}

1. Bayles, M.E.: Designing Online Banner Advertisements: Should We Animate? In: Proceedings of the SIGCHI Conference on Human Factors in Computing Systems: Changing Our World, Changing Ourselves, pp. 363-366. ACM Press, New York (2002)

2. Burke, M., Gorman, N., Nilsen, E., Hornof, A.: Banner Ads Hinder Visual Search and Are Forgotten. In: Extended abstracts of the 2004 Conference on Human Factors and Computing Systems, pp. 1139-1142. ACM Press, New York (2004)

3. Burke, M., Hornof, A., Nilsen, E., Gorman, N.: High-Cost Banner Blindness: Ads Increase Perceived Workload, Hinder Visual Search, and Are Forgotten. In: TOCHI, vol. 12(4), pp. 423-445 (2005) 
4. Faraday, P., Sutcliffe, A.G.: An Empirical Study of Attending and Comprehending Multimedia Presentations. In: Proceedings of 4th Multimedia Conference, pp. 265-275. ACM Press, New York (1996)

5. Faraday, P., Sutcliffe, A.G.: Providing Advice for Multimedia Designers. In: Proceedings of the Conference on Human Factors in Computing Systems, pp. 124-131. ACM Press, New York (1998)

6. Guan, Z., Lee, S., Cuddihy, E., Ramey, J.: The Validity of the Stimulated Retrospective Think-Aloud Method As Measured by Eye Tracking. In: CHI 2006. Proceedings of 2006 Conference on Human Factors in Computing Systems, pp. 1253-1262. ACM Press, New York (2006)

7. Halverson, T., Hornof, A.J.: Strategy Shifts in Mixed Density Search. In: Proceedings of the 48th Annual Meeting of the Human Factors and Ergonomics Society, pp. 1860-1864 (2004)

8. Halverson, T., Hornof, A.J.: Explaining Eye Movements in the Visual Search of Varying Density Layouts. In: Proceedings of the Sixth International Conference on Cognitive Modeling, pp. 124-129 (2004)

9. Heer, J., Chi, C.H.: Separating the Swarm: Categorization Methods for User Sessions on the Web. In: Proceedings of the Conference on Human Factors in Computing Systems, pp. 243-250. ACM Press, New York (2002)

10. Hornof, A.J.: Cognitive Strategies for the Visual Search of Hierarchical Computer Displays. Journal of Human Computer Interaction 19(3), 183-223 (2003)

11. Itti, L., Koch, C.: Computational Modelling of Visual Attention. Nature 2(3), 194-203 (2001)

12. Johansen, S.A., Hansen, J.P.: Do We Need Eye Trackers to Tell Where People Look? In: CHI 2006. Proceedings of Conference on Human Factors in Computing Systems, pp. 923 928. ACM Press, New York (2006)

13. Nielsen, J.: F-Shaped Pattern for Reading Web Content, http://www.useit.com/alertbox/ reading_pattern.html

14. Outing, S., Ruel, L.: The Best of Eye track III: What We Saw When We Looked Through Their Eyes (2004), http://www.poynterextra.org/eye track, /main.htm

15. Pan, B., Hembrooke, H.A., Gay, G.K., Granka, L.A., Feusner, M.K., Newman, J.K.: The Determinants of Web Page Viewing Behavior: An Eye-Tracking Study. In: Proceedings of the 2004 Symposium on Eye Tracking Research \& Applications, pp. 147-154. ACM Press, New York (2004)

16. Reeves, B., Nass, C.: The Media Equation: How People Treat Computers, Television, and New Media Like Real People and Places. Cambridge University Press, Stanford, California (1996)

17. Rauterberg, M., Cachin, C.: Locating the primary attention focus of the user. In: Grechenig, T., Tscheligi, M. (eds.) VCHCI 1993. LNCS, vol. 733, pp. 129-140. Springer, Heidelberg (1993)

18. Zhang, P.: The Effects of Animation on Information Seeking Performance on the World Wide Web: Securing Attention or Interfering with Primary Tasks? Journal of the Association for Information Systems 1(1), 1-28 (2000) 